Pohl, Martin; Nuber, Thomas; Abratis, Joachim; Peschken, Gabriele Düsenstrahlpfähle für die Schleuse Brunsbüttel unter besonderer Berücksichtigung der GW-Verhältnisse

Originalveröffentlichung / Original Publication:

https://doi.org/10.1002/bate.202000036

Verfügbar unter / Available at:

https://hdl.handle.net/20.500.11970/108244

Vorgeschlagene Zitierweise / Suggested citation:

Pohl, Martin; Nuber, Thomas; Abratis, Joachim; Peschken, Gabriele (2020): Düsenstrahlpfähle für die Schleuse Brunsbüttel unter besonderer Berücksichtigung der GW-Verhältnisse. In: Bautechnik 97 (6). S. 427-432. https://doi.org/10.1002/bate.202000036. 


\section{Düsenstrahlpfähle für die Schleuse Brunsbüttel unter besonderer Berücksichtigung der GW-Verhältnisse}

\begin{abstract}
Bei Europas derzeit größter Wasserbaustelle, dem Neubau der fünften Schleusenkammer in Brunsbüttel, kommen zur Rückverankerung der Schleusenkammerwände und -sohle sog. Düsenstrahlpfähle zum Einsatz. Der Einsatz dieser Elemente wurde aufgrund mehrerer Aspekte erforderlich: im Nahbereich der über 100 Jahre alten Schleusen darf nicht gerammt werden, das Grundwasser (GW) weist erhöhte Konzentrationen an Ammonium und Sulfat im Lastabtragungsbereich der geplanten Rückverankerung auf, zudem müssen vergleichsweise hohe Lasten bis zu $2600 \mathrm{kN}$ abgetragen werden.

Die Düsenstrahlpfähle bestehen aus einer Düsenstrahlsäule mit einem Durchmesser von 1,0 m und Längen bis zu 7,5 m, in die ein Stahlzugglied (Durchmesser bis $14 \mathrm{~cm}$ ) eingestellt wird. Da für derartige Düsenstrahlpfähle keine allgemeine bauaufsichtliche Zulassung existiert, wurde nach umfangreichen Untersuchungen und theoretischen Betrachtungen eine Zustimmung im Einzelfall erteilt.

Die Düsenstrahlpfähle wurden gewählt, weil beim Aufdüsen des Baugrunds durch unterschiedliche Erosionswiderstände eine Verzahnung der Düsenstrahlsäule mit dem Baugrund entsteht. Somit wird, unter Einsatz eines Zements mit einem geringen w/z-Wert von 0,6, eine dauerhafte Lastabtragung, selbst bei Betonangriff, gewährleistet. Die Düsenstrahlpfähle kommen in Tiefen ab $25 \mathrm{~m}$ u. GOK (in den tragfähigen Sanden) zum Einsatz. Umfassende Betrachtungen zu den Grundwasserverhältnissen und zur -beschaffenheit konnten den Einsatz der Düsenstrahlpfähle optimieren.
\end{abstract}

Stichworte Düsenstrahlpfahl; ZiE; Schleuse; Grundwasserbeschaffenheit; Betonkorrosion

\section{$1 \quad$ Veranlassung}

Der Nord-Ostsee-Kanal (NOK) durchquert auf knapp $100 \mathrm{~km}$ Länge Deutschlands nördlichstes Bundesland und verbindet die Nordsee mit der Ostsee für seegängige Schiffe. Der NOK gilt als die meistbefahrene künstliche Seewasserstraße der Welt. Die Wasserstandsschwankungen zwischen dem NOK sowie der Nordsee (Elbe) bzw. der Ostsee (Kieler Förde) werden durch Schleusenanlagen in Brunsbüttel und Kiel-Holtenau für die Schifffahrt ausgeglichen. An beiden Standorten bestehen die Schleusenanlagen aus einer „Kleinen Doppelschleuse“ und einer "Großen Doppelschleuse“. Der NOK und die kleinen Schleusen wurden im Jahr 1895 nach acht Jahren Bauzeit in Betrieb genommen. Der erste Ausbau des NOK erfolgte bereits von 1907 bis 1914. In diesem Zuge wurden auch die großen Schleusen errichtet. Die Schleusen sind seitdem durchgehend in Betrieb.
Jet grouted piles for the Brunsbüttel lock with special consideration of the GW-conditions

At Europe's currently largest water engineering construction site, the construction of the fifth lock chamber in Brunsbüttel, so-called jet grouted piles are used to anchor back the lock chamber walls and lock base. The use of these elements became necessary for several reasons: in the vicinity of the locks, which are over 100 years old, driving is not permitted, groundwater that attacks concrete (ammonium and sulphate) occurs in relevant proportions in the load transfer area and comparatively high loads of up to $2600 \mathrm{kN}$ must be transferred.

The jet grouted piles consist of a jet column with a diameter of $1.0 \mathrm{~m}$ and lengths up to $7.5 \mathrm{~m}$, into which a steel tension member (diameter up to $14 \mathrm{~cm}$ ) is inserted. As there is no general approval by the building authorities for such jet grouted piles, the Federal Ministry of Transport and Digital Infrastructure (BMVI) granted approval in individual cases (ZiE) after extensive investigations and theoretical considerations.

The jet grouted piles were chosen because the jet grouting of the subsoil creates an interlocking of the jet grout column with the subsoil due to different erosion resistances. Thus, using a cement with a low w/c-value of 0.6 , a permanent load transfer is guaranteed, even if the concrete is attacked. The jet grouted piles are used at depths from $25 \mathrm{~m}$ below ground level (in the load-bearing sands). Comprehensive observations of the groundwater conditions and the nature of the groundwater were utilized to optimise the use of the jet grouted piles.

Keywords jet grouted pile; lock; groundwater quality; concrete corrosion; ammonium

Die kleinen Schleusen in Brunsbüttel wurden bereits in den 1980er-Jahren saniert. Die großen Schleusen bedürfen nach über 100 Jahren Nutzungszeit ebenfalls einer Grundinstandsetzung des Massivbaus, Stahlwasserbaus sowie der maschinen- und elektrotechnischen Anlagen zur Aufrechterhaltung der Verkehrs- und Betriebssicherheit.

Aufgrund der Art der erforderlichen Sanierungsarbeiten ergäben sich zwangsläufige Sperrzeiten für die Schifffahrt, die als nicht tolerabel beurteilt wurden. Um die Schleusenverfügbarkeit nicht einzuschränken, ist der Bau einer weiteren großen Schleusenkammer, der sog. fünften Kammer, erforderlich, sodass der Schifffahrt zwei große Schleusen zur Verfügung stehen. Für die Lage der fünften Kammer wurden verschiedene Varianten untersucht. Als Kriterien wurden Aspekte wie Nautik, Grundstücksgrenzen, bestehende Bebauung herangezogen. Die NutzenKosten-Untersuchung ergab den höchsten Nutzwert bei 


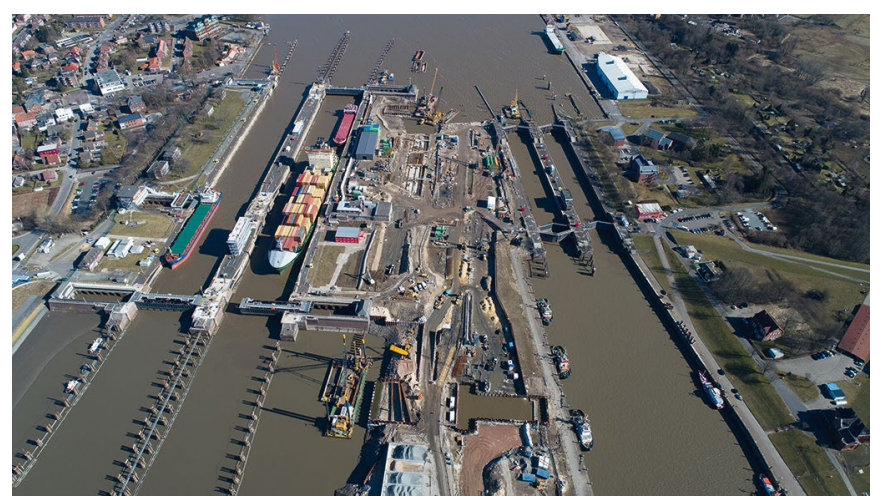

Bild 1 Baustelle der fünften Schleusenkammer auf der Schleuseninsel (Jahr 2018)

Construction site of the $5^{\text {th }}$ lock chamber between the existing locks (year 2018)

einer Grundinstandsetzung der Großen Schleuse mit einem vorgezogenen Neubau einer fünften Schleusenkammer auf der Schleuseninsel, d.h. zwischen der Großen Schleuse im Norden (Bild 1, links) und der Kleinen Schleuse im Süden (Bild 1, rechts).

\section{Baugrund}

Zur Erkundung des Baugrunds wurden rd. 2100 m Bodenproben erbohrt und im geotechnischen Labor umfassend untersucht. Der Baugrundaufbau im Bereich der Schleusenanlage Brunsbüttel kennzeichnet sich durch eiszeitliche Ablagerungen in größeren Tiefenbereichen ab etwa $35 \mathrm{~m}$ unter Geländeoberkante (GOK) sowie darüber befindlichen jüngeren Ablagerungen infolge des nacheiszeitlichen Meeresspiegelanstiegs (Bild 2). Im Konkreten steht von der GOK bis in eine Tiefe von etwa $25 \mathrm{~m}$ weicher Klei an. Der Klei besteht aus einer vertikalen wieder- holenden Abfolge von Ton, Schluff und Feinsand in unterschiedlicher Ausprägung. Unterlagernd folgen gut tragfähige Sande mit Kiesanteilen. In dieser Bodenschicht gibt es eine Abfolge mit eiszeitlichen bindigen Ablagerungen in Form von Beckenton und Geschiebemergel; überwiegend fester Konsistenz. Durch periglaziale Prozesse ist der Geschiebemergel lokal stark aufgearbeitet und mit Sand durchsetzt, sodass kleinräumige Änderungen auftreten.

Beim Laborprogramm sind besonders die ausgeführten Triaxial- und Ödometerversuche an bindigen Bodenproben zur Herleitung realitätsnaher Bodenkennwerte für die Bemessung der Bauteile sowie des spannungsabhängigen Tragfähigkeitsverhaltens hervorzuheben. Eine Aufgliederung und detaillierte Betrachtung der Versuchsergebnisse am Klei in die prägenden sandigen, schluffigen und tonigen Eigenschaften ermöglichten, das Bodenverhalten technisch zutreffend sowie wirtschaftlich optimiert zu erfassen [1]. Für die Verwendung des Aushubmaterials für Deichschutzmaßnahmen war eine Unterteilung in sandigen und tonigen Klei relevant. Hierfür wurde der Klei gemäß [2] weitergehend untersucht und bewertet.

\section{Einfluss der Nachbarbebauung auf Planung und Herstellung}

Unter Berücksichtigung des Baugrundaufbaus wurden die Auswirkungen von Rammerschütterungen beim geplanten Neubau der fünften Schleusenkammer betrachtet. Dabei waren besonders die in unmittelbarer Nähe liegenden alten und bautechnisch sehr empfindlichen Schleusen zu berücksichtigen, da deren Betrieb ohne Einschränkungen durchgehend gewährleistet sein muss. Im Ergebnis der Untersuchungen dürfen in weiten Bereichen der geplanten fünften Kammer nur erschütterungsarme,

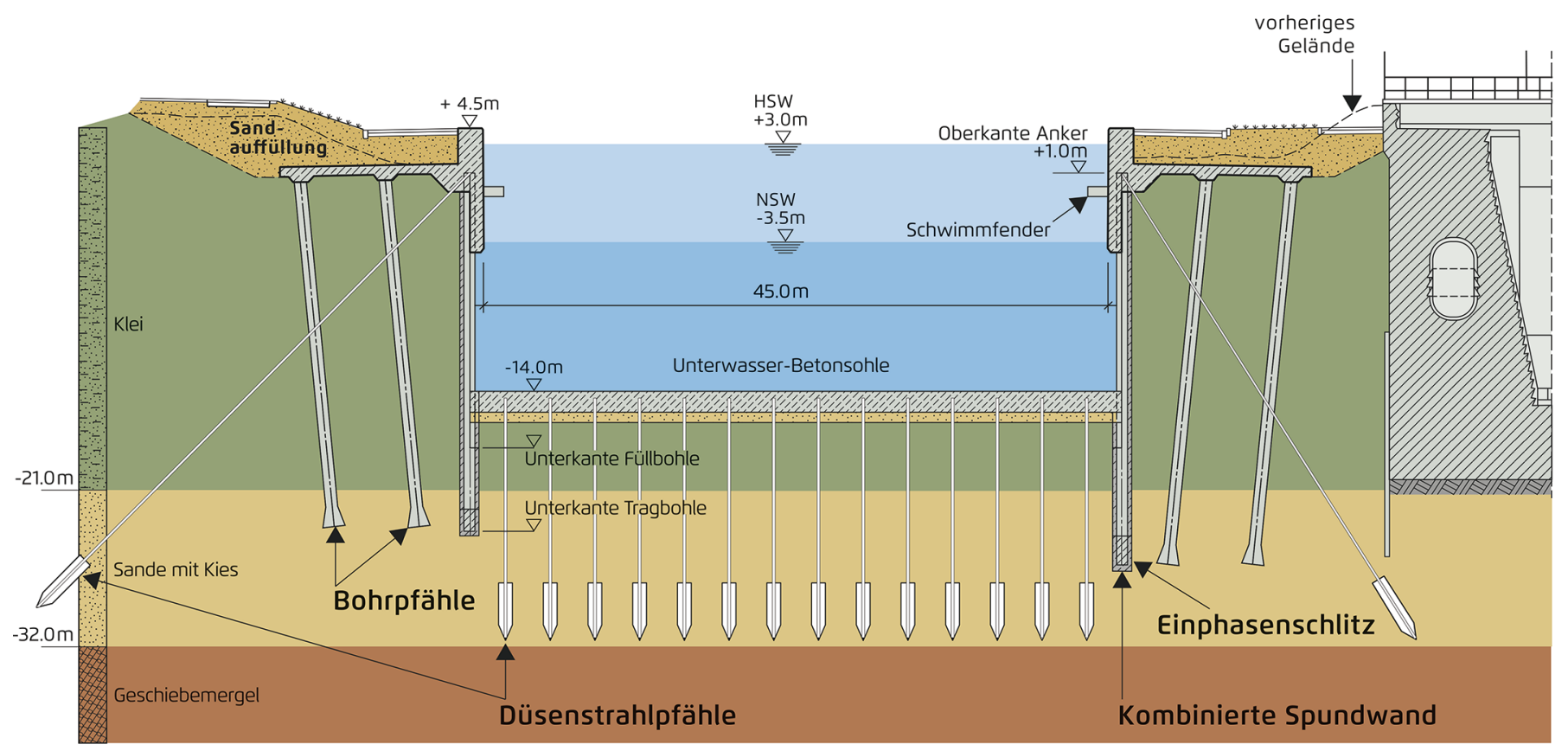

Bild 2 Querschnitt der Schleusenkammer (schematisch) Cross section of the lock chamber (schematic) 
d.h. greifende oder bohrende Bauverfahren eingesetzt werden. Das Einbringen von Stahlprofilen mittels Schlagoder Vibrationsrammung schied somit aus.

Bei der Wandherstellung entschied man sich für die Herstellung einer gegreiferten Dichtwand, in die Stahlprofile als kombinierte Spundwand eingestellt werden (Bild 2). Aus der Bemessung der Kammerwände ergaben sich Rückverankerungskräfte bis zu $2600 \mathrm{kN}$. Weil für die Rückverankerung nur bohrende Verfahren in Betracht kamen, entschied man sich für die Herstellung sog. Düsenstrahlpfähle (Abschn. 5). Erfahrungen mit dieser Art von Rückverankerungselementen liegen im übertragenen Sinne z. B. mit [3, 4] vor.

\section{4}

\section{Grundwasserverhältnisse}

Zur Erfassung der Grundwasserdynamik, zur Festlegung der für die Bemessung der Schleuse anzugebenden charakteristischen Grundwasserstände sowie für die erforderliche Einstufung der Grundwasserbeschaffenheit hinsichtlich der Betonaggressivität nach DIN 4030 [5] wurde ein flächendeckendes Grundwassermessnetz errichtet. Zum einen wurden dafür Porenwasserdruckmessgeber in ausgewählten Bohrungen eingebaut und zum anderen „klassische“ Grundwassermessstellen errichtet, die z.T. in Gruppen angeordnet sind, die aus mehreren Messstellen mit Filterstrecken innerhalb verschiedener Tiefenlagen bestehen. Somit ist eine räumlich differenzierte und tiefenorientierte Bewertung der Grundwasserverhältnisse im Untersuchungsgebiet möglich.

Für die Sande mit Kies zeigen die Grundwasserstandsmessungen gegenüber den tidebeeinflussten Wasserständen der Elbe einen leicht gedämpften und zeitverzögerten Verlauf der Grundwasserpotenziale. Dabei entsprechen die mittleren Grundwasserpotenziale nahezu dem mittleren Wasserstand der Elbe. Im darüber befindlichen oberflächennah anstehenden Klei ist dagegen nur ein untergeordneter Einfluss der Tide zu erkennen. Dort werden die Grundwasserpotenziale im Wesentlichen von meteorologischen Ereignissen dominiert [6].

Um die Grundwasserbeschaffenheit - neben der Einschätzung hinsichtlich der Betonaggressivität - umfassender bewerten zu können, erfolgte eine Analytik mit einem Parameterumfang, der sämtliche Hauptionen einschließt und somit deutlich über die nach [5] erforderlichen Parameter hinausgeht. Neben einer Plausibilisierung der Analyseergebnisse durch eine Ionenbilanz nach [7] ist somit auch eine Klassifizierung der Grundwassertypen nach [8] sowie die Bestimmung des sog. Basenaustauschindex (BEX) nach [9] möglich, mit dessen Hilfe abgeschätzt werden kann, inwieweit Grundwässer durch Versalzungsbzw. Aussüßungsprozesse beeinflusst sind. Der BEX wird anhand der Konzentration der salzwasserbürtigen Kationen Natrium, Kalium und Magnesium und des Chloridgehalts ermittelt. Bei negativen BEX-Werten unterliegt das Grundwasser einer Versalzung, wohingegen Grundwäs- ser, die Aussüßungprozessen unterliegen, durch positive Werte gekennzeichnet sind. Eine Bewertung, ob das Grundwasser Versalzungs- bzw. Aussüßungsprozessen unterliegt, ist bei einer Betrachtung der Grundwasserbeschaffenheit im Küstenbereich maßgeblich, da eine Infiltration von salzhaltigem Oberflächenwasser in einen Grundwasserleiter die wesentliche Einflussgröße auf den gesamten Grundwasserchemismus darstellt.

Für die Bewertung hinsichtlich der Betonaggressivität nach [5] zeigten sich die Parameterwerte für die Wasserinhaltsstoffe Ammonium und Sulfat als besonders relevant. Hohe Ammoniumkonzentrationen im Grundwasser sind zumeist auf anthropogene Quellen wie z.B. Abwässer zurückzuführen. Hohe geogen bedingte Ammoniumkonzentrationen - wie sie hier $\mathrm{zu}$ vermuten sind - können bisher nur in den norddeutschen Marschgebieten beobachtet werden, wie sie z.B. in [10] bei Untersuchungen in der Hamburger Elbmarsch beschrieben sind.

Der für die Sande anhand tidebereinigter Grundwasserstandsmessungen ermittelte Grundwassergleichenplan gemäß [11] sowie die Analyseergebnisse für die Parameter Ammonium und Sulfat sowie des BEX sind für den Lastabtragungsbereich der Verankerung in Bild 3 dargestellt.

Für diesen Grundwasserhorizont ist ein schwach angreifender Betonangriff (XA1) durch Sulfat (treibender Angriff) und Ammonium (lösender Angriff) anzunehmen. Die gemessenen Werte innerhalb des Lastabtragungsbereichs liegen für SO4 zwischen 230 und $340 \mathrm{mg} / \mathrm{l}$, für NH4 zwischen 16 und $25 \mathrm{mg} / \mathrm{l}$.

Die vergleichsweise geringe Ammoniumkonzentration im elbseitigen Bereich ist auf einen stärkeren elbbürtigen Salzwassereinfluss zurückzuführen, was anhand der negativen BEX-Werte belegt werden kann. Diese Messstelle liegt im südwestlichen Bereich der Schleuseninsel, der von der nordwestlichen Seite wie auch von der südöstlichen Seite von der Elbe umfasst wird. Durch diese Lage unterliegt die Grundwasserbeschaffenheit einem - im Vergleich zum nordöstlichen Bereich der Schleuseninsel - verstärkten Elbwassereinfluss. Diese Beobachtungen decken sich zudem mit dem Strömungsbild. Der nordöstliche Bereich der Schleuseninsel wird landseitig angeströmt. Das Grundwasser entlastet in Richtung der beiden elbseitigen Vorhäfen. Auf dem südwestlichen Teil der Schleuseninsel deutet sich eine Grundwasserkuppe an, die sich ebenfalls in Richtung der beiden Vorhäfen hin entlastet. Neben den räumlichen Unterschieden zeigt sich ein deutliches tiefenabhängiges Konzentrationsgefälle der einzelnen Parameter (Bild 4).

Die Grundwässer, die aus den Filterstrecken im Klei entnommen wurden, lassen sich zudem aufgrund der Ionenverhältnisse von den Grundwässern, die aus den tiefer liegenden Sanden entnommen wurden, unterscheiden. In der Kleischicht liegen erdalkalisch bzw. alkalisch geprägte Grundwässer vor, die z.T. durch hohe Sulfat- und Eisengehalte gekennzeichnet sind. Die BEX-Werte $>0$ zei- 


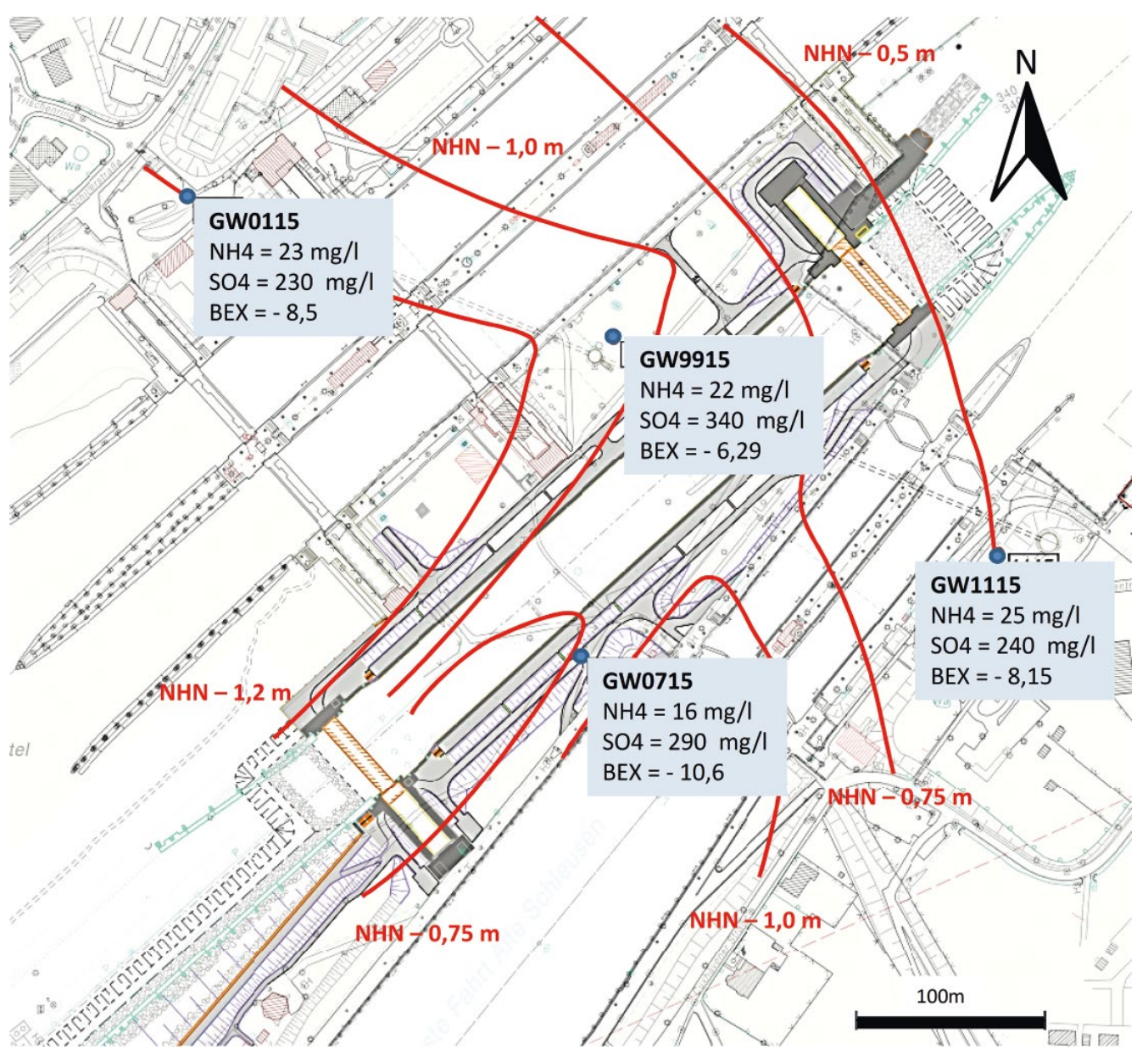

Bild 3 Grundwassergleichenplan mit Stoffkonzentrationen (Istzustand) Goundwater level plan with substance concentration (present state)

gen, dass die Wässer keinen Versalzungsprozessen unterliegen.

Wenige dm unterhalb der Kleischicht wurden bis zu $20 \mathrm{mg} / \mathrm{l}$ höhere Ammoniumkonzentrationen als im tiefer liegenden Verankerungsbereich festgestellt. Eine solche Tiefenabhängigkeit der Ammoniumkonzentration bei ähnlichen hydrogeologischen Verhältnissen wurde bereits bei [12-15] beschrieben und ist als plausibel zu werten. Die Tiefenabhängigkeit der Ammomiumkonzentration sowie die Lokation der höchsten Konzentrationen unterhalb der Kleischichten lassen die organischen Kleischichten als Ammoniumquelle vermuten, wie auch Untersuchungen von [10] gezeigt haben.

Insgesamt lässt sich zusammenfassen, dass die Grundwasserbeschaffenheit des nordöstlichen Bereichs überwiegend landseitigen Einflüssen unterliegt. Im südwestlichen Bereich der Schleuseninsel liegt ein Austausch mit den elbseitigen Vorhäfen der bestehenden Schleusen vor. Es ist anzunehmen, dass die Abgrabungen der Schleuseninsel zum Bau des elbseitigen Vorhafens und die damit verbundene verstärkte Infiltration von salzhaltigem Elbwassser zu einer Verringerung der Ammoniumkonzentrationen führen wird. Bereits heute kann für den elbseitigen Schleusenbereich eine nicht betonangreifende Ammoniumkonzentration (gemäß [5]) angesetzt werden, sodass in diesem Bereich geringere Anforderungen an die Düsenstrahlpfähle gelten.

Während dem Sulfatangriff mit einem HS-Zement begegnet werden kann, bestehen für den Ammoniumangriff keine betontechnologischen Begegnungsmöglichkeiten. Dem aktuellen technischen Stand, d.h. [5] folgend, wurden die Konsequenzen für das Rückverankerungselement berücksichtigt (Abschn. 5). Untersuchungen von [16] legen jedoch nahe, dass erst bei weitaus höheren Ammoniumkonzentrationen Auswirkungen für einen Beton zu erwarten sind. Die aus den GW-Analysen hergeleiteten Eigenschaften für die Düsenstrahlpfähle können folglich als weit auf der sicheren Seite liegend angesehen werden, was für ein Bauwerk mit geplanten 100 Jahren Nutzungszeit aber dennoch als gerechtfertigt angesehen werden kann.

\section{$5 \quad$ Düsenstrahlpfahl zur Rückverankerung}

Beim Düsenstrahlverfahren wird der Boden mit einem Schneidstrahl aufgeschnitten und mit Zement versetzt. Von besonderer Bedeutung für das Ergebnis der somit herzustellenden Düsenstrahlsäule sind neben dem Wasser-Zementgehalt und der Zieh- und Rotationsgeschwindigkeit die an der Düsöffnung herrschenden Drücke 
GW01

O7F $07 \mathrm{~T} 15$

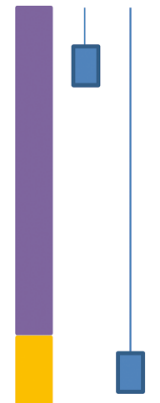

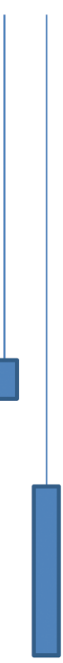

$\mathrm{NH} 4=18 \mathrm{mg} /$ $\mathrm{SO} 4=280 \mathrm{mg} /$ $\mathrm{BEX}=+16,25$

\section{$\mathrm{NH} 4=30 \mathrm{mg} / \mathrm{l}$ $\mathrm{SO} 4=41 \mathrm{mg} / \mathrm{l}$ $\mathrm{BEX}=+4,65$ $\mathrm{SO} 4=230 \mathrm{mg} /$ $\mathrm{BEX}=-8,50$}

GW07

O7F $07 T 15$

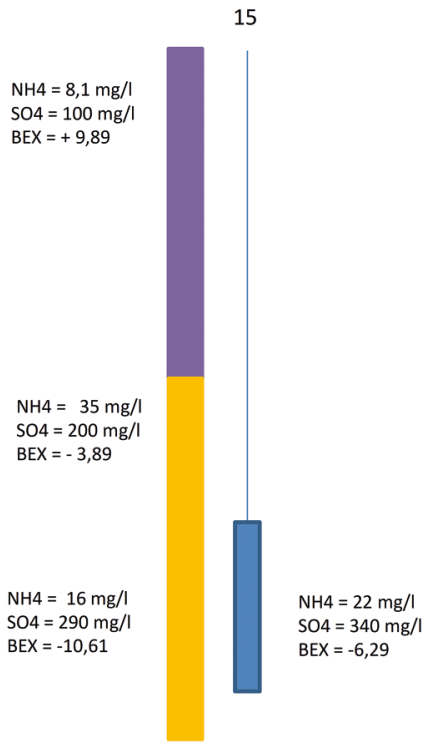

GW11

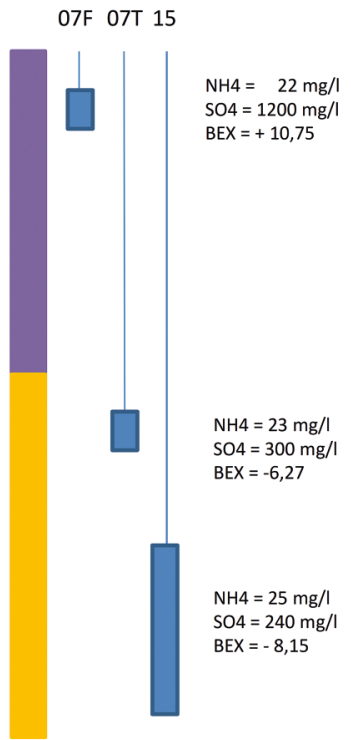

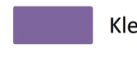

Bild 4 sowie auch die Durchmesser der Düsöffnungen, deren Anordnung und Verschleiß [1, 17-21].

Durch Inhomogenitäten im Baugrund, wie Lagerungsdichte und Kornfraktion, ergibt sich eine unterschiedliche Eindringtiefe für den Schneidstrahl. Hieraus resultiert eine Düsenstrahlsäule mit keiner rein zylindrischen Form, sondern mit gewissen Einbuchtungen und Auskragungen. Dieses Verhalten macht man sich für die dauerhafte Rückverankerung unter betonangreifendem Ammonium zunutze.

Bei einem verpressten, annähernd ebenförmig zylindrischen Rückverankerungselement würde der Verspannungsdruck und damit die Mantelreibung durch bereits geringe Betonkorrosion infolge lösenden Angriffs abfallen [22] und das Bauteil versagen. Die unregelmäßige Verzahnung der Düsenstrahlsäule mit dem Baugrund gewährleistet hingegen eine Kraftübertragung über Druckkräfte auch bei einer prognostizierten Betonkorrosionszone von $1 \mathrm{~mm} / 100$ Jahre.

Dementsprechend wurden für die fünfte Kammer die sog. Düsenstrahlpfähle als Rückverankerungselemente geplant. Diese bestehen aus einer Düsenstrahlsäule mit einem Durchmesser von 1,0 $\mathrm{m}$ und Längen bis zu 7,5 m, in die ein Stahlzugglied (Durchmesser bis $14 \mathrm{~cm}$ ) eingestellt wird (s. Bild 2). Es ist also ein „Pfahl“ mit begrenzter Krafteinleitungslänge. Da eine solche Art der Rückerverankerung kein übliches und daher auch kein allgemein bauaufsichtlich zugelassenes Verfahren ist, wurden einzuhaltende Kriterien zur Gewährleistung eines dauerhaft hoch tragfähigen Düsenstrahlpfahls für die geplante Nutzungsdauer von 100 Jahren definiert. Diese mündeten in einer „Zustimmung im Einzelfall“ (ZiE), welche durch das Bundesministerium für Verkehr und digitale Infrastruktur (BMVI) erlassen wurde. Die kennzeichnenden Eigenschaften der herzustellenden Düsenstrahlpfähle sind: $w / z=0,6$, Mindestfestigkeitsklasse C25/30, Mindestzementgehalt $280 \mathrm{~kg} / \mathrm{m}^{2}$, Durchmesser $\geq 1,0 \mathrm{~m} \mathrm{[1]}$ sowie eine Wendelgeometrie in Anlehnung an bestehende allgemeine bauaufsichtliche Zulassungen.

Die initial angenommenen Herstellparameter hatten $\mathrm{w} / \mathrm{z}$ Werte von 0,5. Da solch geringe w/z-Werte beim Düsenstrahlverfahren nicht üblich sind, wurde die Machbarkeit der Herstellung der Düsenstrahlpfähle im Vorwege der eigentlichen Baumaßnahme umfassend untersucht. Für die eingesetzte Gerätekonstellation der Baufirma wurden die Herstellparameter für eine Düsenstrahlsäule mit den o. g. Eigenschaften erfolgreich ermittelt. Gleiches geschah nochmals für die Gerätekonstellation der ausführenden Baufirma bei der eigentlichen Baumaßnahme [1].

Die Tragfähigkeit der Düsenstrahlpfähle wurde im Vorwege sowie im Rahmen der eigentlichen Baumaßnahme mittels Probebelastungen nachgewiesen. Bei einer aufgebrachten max. Zugkraft von $4300 \mathrm{kN}$ betrug die bleibende Verformung lediglich $3 \mathrm{~cm}$. Aufgrund der hohen Anforderungen an das Bauteil werden zudem an jedem Düsenstrahlpfahl Abnahmeprüfungen durchgeführt. Bei allen bis dato hergestellten Düsenstrahlpfählen erfüllen die Untersuchungsergebnisse die Anforderungen; hierzu zählen auch die weiterhin stattfindenden betontechnologischen Untersuchungen an ergänzenden Blindsäulen.

Da der Zementverbrauch mit etwa 2,9 t/lfd. m vergleichsweise hoch ist, wurden weitergehende Untersuchungen 
angestellt, den Ressourceneinsatz zu reduzieren. Durch eine Variation der Herstellparameter, d.h. Düsdrücke, Rotations- und Ziehgeschwindigkeiten (in unterschiedlichen Herstellungsphasen), sowie des w/z-Werts konnte der theoretisch erforderliche Zementverbrauch um bis zu $40 \%$ gesenkt werden. Eine finale Verifikation steht jedoch noch aus.

\section{Literatur}

[1] Pohl, M.; Abratis, J. (2020) Planung und Bau der 5. Schleusenkammer in Brunsbüttel in: Mitteilungen des Institutes und der Versuchsanstalt für Geotechnik der Technischen Universität Darmstadt, H. 110.

[2] EAK (2002) Empfehlungen für die Ausführung von Küstenschutzwerken in: Die Küste, H. 65 (korrigierte Ausgabe 2007).

[3] Köster, H.; Dietz, K. (2006) Herstellung von Zuggliedern in aggressivem Grundwasser in: DGGT [Hrsg.] Vorträge der Baugrundtagung 2006 - Tagungsband zur 29. Baugrundtagung der Deutschen Gesellschaft für Geotechnik e.V. (DGGT) mit Fachausstellung „Geotechnik“. Bremen, 27.-30. Sept. 2006.

[4] Bautechnik aktuell (2000) Kajeninstandsetzung im Marinearsenal Kiel in: Bautechnik 77, H. 9, S. 704.

[5] DIN 4030-1 (2008) Beurteilung betonangreifender Wässer, Böden und Gase - Teil 1: Grundlagen und Grenzwerte. Berlin: Beuth-Verlag.

[6] Schulze, P.; Pohl, M. (2011) Porenwasserdruckmessungen für den Seeschleusenneubau Brunsbüttel in: BAW [Hrsg.] BAWKolloquium - Tagungsband Geohydraulische Erkundungsverfahren. Hannover, 22. Nov. 2011.

[7] DWA (2011) Arbeitsblatt DWA-A-909: Grundsätze der Grundwasser-Probennahme. ATV.

[8] Furtak, H.; Langguth, H. R. (1967) Zur Hydrochemischen Kennzeichnung von Grundwässern und Grundwassertypen mittels Kennzahlen in: Mem. IAH-Congress, VII. Hannover, 1965. S. 86-96.

[9] Stuyfzand, P. J. (1989) A new hydrochemical classification of Water Types in: IAHS-Publication 182, pp. 89-98.

[10] Gröngroft, A. (1992) Untersuchung des Sickerwasser- und Stoffeintrags aus Hafenschlick-Spülfeldern in den oberen Grundwasserleiter der Hamburger Elbmarsch in: Verein zur Förderung der Bodenkunde in Hamburg. Hamburg.

[11] Serfes, M. E. (1991) Determining the mean hydraulic gradient of groundwater affected by tidal fluctuations in: Ground Water 29, no. 4, pp. 549-555.
[12] Jiao, J.; et al. (2010) Abnormally high ammonium of natural origin in a coastal aquifer-aquitard system in the Pearl River Delta, Chin. in: Environmental Science \& Technology 44, no. 19, pp. 7470-7475.

[13] Lindenbaum, J. (2012) Identification of sources of ammonium in groundwater using stable nitrogen and boron isotopes in Nam Du. Hanoi [Master-Thesis]. Department of Geology, Lund University, Schweden.

[14] Glessner, J. J. G.; Roy, W. R. (2009) Paleosols in central Illinois as potential sources of ammonium in groundwater in: Ground Water Monit. Rem. 29, no. 4, pp. 56-64.

[15] Schilling, K. E. (2002) Occurrence and distribution of ammonium in Iowa groundwater in: Water Environ. Res. 74, no. 2 , pp. $177-186$

[16] Rechenberg, W.; Sylla, H.-M. (1993) Die Wirkung von Ammonium auf Beton in: Beton 1, S. 26-31.

[17] Sondermann, W. (2012) Die Reichweite des Schneidstrahls beim Düsenstrahlverfahren - Einflussfaktoren in: DGGT [Hrsg.] Vorträge 32. Baugrundtagung. Mainz, 26.-29. Sept. 2012.

[18] Krentz, M. (2015) Zur guten fachlichen Praxis des Düsenstrahlverfahrens in: Geotechnik 38, H. 1, S. 56-64.

[19] Bergschneider, B. (2002) Zur Reichweite beim Düsenstrahlverfahren im Sand [Dissertation]. Bergische Universität Wuppertal, Fachbereich Bauingenieurwesen, Bodenmechanik und Grundbau, Bericht 24.

[20] Kummerer, C.; Holl, T.; Sigmund, C. (2016) Aktuelle Entwicklungen zur Reichweitenoptimierung und Qualitätskontrolle beim Soilcrete-Düsenstrahlverfahren unter schwierigen Bodenverhältnissen in: DGGT [Hrsg.] Vorträge 34. Baugrundtagung. Bielefeld, 14.-17. Sept. 2016.

[21] Kayser, J.; Odenwald, B.; Oltmanns, W. (2007) Herstellung der Baugrube für Schleuse Uelzen II mit dichtender Düsenstrahlsohle in: BAW; TU Dresden [Hrsg.] Tagungsband Johann-Ohde-Kolloquium. Hannover, 15. Nov. 2007.

[22] Domes, X. (2015) Cement grouting during installation of ground anchors in non-cohesive soils [Dissertation]. Norwegian University of Science and Technology, Department of Civil and Transport Engineering.

Dipl.-Ing. Gabriele Peschken gabriele.peschken@bmvi.bund.de Bundesministerium für Verkehr und digitale Infrastruktur Referat WS 12 - Technik der Wasserstraßeninfrastruktur Robert-Schuman-Platz 1

53175 Bonn

Dr.-Ing. Thomas Nuber thomas.nuber@baw.de Bundesanstalt für Wasserbau

Referat Geotechnik Nord

Wedeler Landstraße 157

22559 Hamburg

Dipl.-Ing. Joachim Abratis

joachim.abratis@wsv.bund.de

Wasserstraßen- und Schifffahrtsamt Brunsbüttel

Alte Zentrale 4

25541 Brunsbütte

\section{Zitieren Sie diesen Beitrag}

Pohl, M.; Nuber, T.; Abratis, J.; Peschken, G. (2020) Düsenstrahlpfähle für die Schleuse Brunsbüttel unter besonderer Berücksichtigung der GW-Verhältnisse. Bautechnik 97, H. 6, S. 427-432.

https://doi.org/10.1002/bate.202000036 